\title{
Comparison of Combustion Dynamic Characteristics of Two Advanced Multi-Cup Fuel Injectors
}

\author{
Waldo A. Acosta ${ }^{1}$ \\ Army Research Laboratory, Cleveland, $\mathrm{OH}$ 44135, USA \\ Clarence T. Chang ${ }^{2}$ \\ NASA Glenn Research Center, Cleveland, OH 44135, USA
}

\begin{abstract}
An experimental investigation of the combustion dynamic characteristics of two advanced multi-cup lean direct injectors (LDI) under simulated gas turbine combustor conditions was conducted. The objective was to gain a better understanding of the physical phenomena inside a pressurized flame tube combustion chamber and study the effects of injector flow number on combustion dynamics. The injectors are known as Three-zone Injectors one and two or 3ZI-1 and 3ZI-2, respectively. The injectors were experimentally evaluated at inlet pressures up to $1.724 \mathrm{MPa}$, non-vitiated air temperatures up to $828 \mathrm{~K}$, and adiabatic flame temperatures up to 1975K. Dynamic pressure measurements were taken upstream of the injectors and in the combustion zone. The combustion dynamic behavior of the two injectors was measured over a range of inlet pressures, inlet temperatures, fuel air ratios, and fuel flow splits.
\end{abstract}

\section{Nomenclature}

$c \quad=$ speed of sound

$f \quad=$ frequency

$L \quad=$ combustor length

$p^{\prime} \quad=$ unsteady pressure

$\mathrm{P} \quad=$ pressure

$\mathrm{T}=$ temperature

\section{Greek alphabet}

$\phi=$ equivalence ratio

$\phi 1 \quad=$ pilot equivalence ratio

$\phi 2=$ center main injectors equivalence ratio

\section{Subscripts}

$3=$ combustor inlet

$4=$ combustor exit
Acronyms
$\mathrm{CO}=$ carbon monoxide
$C R Z=$ central recirculation zone
$E R A=$ Environmentally Responsible Aviation
lbf $\quad=$ pound-force
$L D I=$ lean direct injection
$L P P=$ lean pre-mixed pre-vaporized
$\mathrm{NO}_{\mathrm{x}}=$ nitrogen oxides
$\mathrm{PVC}=$ precessing vortex core
3ZI $=$ three-zone injector

\footnotetext{
${ }^{1}$ Aerospace Engineer, Vehicle Technology Directorate, 21000 Brookpark Rd./MS 5-10, AIAA Senior Member.

${ }^{2}$ Aerospace Engineer, Combustion Branch, 21000 Brookpark Rd./Mail Stop 5-10, Cleveland, OH.
} 


\section{I. Introduction}

$\mathrm{L}$ EAN Direct Injection (LDI) combustion has been shown as a useful lean-burn mode for high-pressure combustion above $6,000 \mathrm{kPa}$ with low emissions [1-3]. At elevated combustor inlet temperature and pressure, the fuel ignition delay time is reduced considerably and the fuel burns while it mixes, and thus called "direct" injection of the fuel into the combustion zone. When multiple, small diameter fuel nozzles are used, it reduces the transport distance and speeds up mixing as well as improves fuel-air mixture homogeneity to reduce hot spots.

Under NASA's Environmentally Responsible Aviation (ERA) Project, Parker Hannifin developed a nextgeneration LDI low-emission fuel injector for a 55:1 pressure ratio gas turbine engine cycle [4]. LDI concepts have the potential to have levels of oxides of nitrogen (NOx) emissions comparable to lean pre-mixed pre-vaporized (LPP) concepts. LDI concepts are very attractive for aviation applications because of their improved flashback and auto ignition avoidance and stability characteristics. But, like LPP concepts, they may also be highly prone to dynamic instabilities or thermoacoustic oscillations under certain operating conditions.

Combustion instabilities occur in highly intense combustors and are the result of the complex interactions between the combustion process and unsteady flow that produces a periodic heat addition process that results in large amplitude oscillations that could span one or more of the combustor acoustic modes. Rayleigh's criterion establishes that to drive a combustion instability, the heat addition process must be in phase with the combustor pressure oscillations [5]. Otherwise, the heat addition oscillations damp the pressure oscillations.

Swirling flows are the most common method used in gas turbine engines to stabilize the flame and promote fuel air mixing. The swirling flow creates a central recirculation zone (CRZ) that anchors the flame and provides stability. In addition, this CRZ is also associated with a precessing vortex core (PVC) where the axis of rotation of the swirling flow rotates around the geometric center of the flow field [6]. Lean-burning swirl stabilized flames have exhibited combustion dynamics that are associated with swirl-acoustic interactions. It has been reported that there is a non-linear interaction between the PVC and the thermo-acoustic modes in the flames of premixed methane-air combustors. Several review papers summarize the efforts conducted over the past few decades aimed at understanding the mechanisms governing flame response [7-9].

Over the past few years, ARL and NASA have conducted various in-house and contractual efforts to understand the combustion dynamics of LDI concepts. Some of those efforts utilized a single injector specifically designed to generate combustion instabilities in a laboratory setting [10-13]. Others utilized multiple injectors more closely resembling an actual engine configuration and were experimentally evaluated at inlet conditions close to actual engine conditions. [14-16].

The work presented here is for an LDI concept specifically designed for a 55:1 pressure ratio gas turbine engine cycle and more representative an actual engine configuration. This work aims to characterize the combustion dynamics behavior of two three-zone fuel injectors at higher inlet pressures $(1,724 \mathrm{kPa})$ and temperatures $(827 \mathrm{~K})$ conditions.

\section{Experimental Setup and Measurements}

\section{A. Experimental Setup}

Experiments were conducted in stand 1 of the CE-5B flame tube test rig at the Glenn Research Center. A schematic showing the location of the injector and selected instrumentation is shown in Fig. 1. The LDI injectors utilized in these tests were developed by Parker Hannifin under NASA's Environmentally Responsible Aviation (ERA) project. The multipoint LDI concept, known as the Three-Zone Injector, or 3ZI, is shown in Fig. 2. The overall design strategy for this injector was a practical injector that could be inserted through a hole in the engine casing of a notional 60,000 lbf thrust engine.

The optimized 3ZI injector utilizes three compact arrays of sprays to produce an effective multipoint LDI design [4]. The injector features five spray cups arranged vertically in each of the three panels. The center panel discharges fuel and air axially into the combustor while the side panels are slightly angled relative to the center panel. This arrangement creates three flame zones with reduced coupling between zones, which lead to improved ignition characteristics and staged low power operation. The direction of the air swirlers alternated from cup to cup in a checkerboard way.

Other improvements from previous LDI multipoint designs include, improved thermal management by reducing the conduction path between the fuel-carrying components and hot external components, and improvements to the spray cup design to allow a greater control of the cup aerodynamics. 
There were four different designs of the 15-cup 3ZI LDI injector with different flow numbers, fuel staging, and atomizer location built. Two of them had the pilot cup in the second row of the center panel, were designed for atmospheric testing, and the location of the fuel tip was also different. The other two were designed for high pressure testing, the pilot was moved to the top spray cup in the center panel, and the number of fuel circuits was reduced to three. One fuel circuits was dedicated to the pilot, the second one was for the other four spray cups below the pilot in the center row, and the third circuit was for the two side rows of spray cups. From now on we will refer to those injectors as 3ZI-1 and 3ZI-2. In addition to the changes noted above, the flow number of the 3ZI-2 was half of the flow number of 3ZI-1. A more detailed discussion on the injector design and features can be found in [4]

\section{B. Measurements}

The combustion dynamic characteristics of the 3ZI-1 and 3ZI-2 injectors were measured over a range of combustor operating conditions. The nominal range of conditions is shown in Table 1. Steady state data was acquired at a rate of $1 \mathrm{~Hz}$ utilizing the NASA Glenn ESCORT data

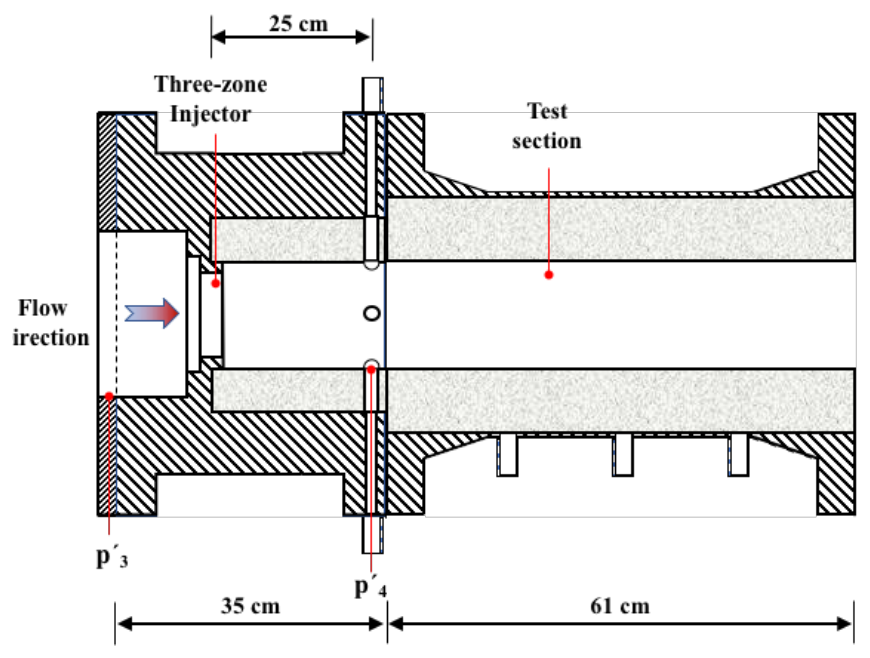

Fig. 1 Schematic of the CE-5B flame tube.
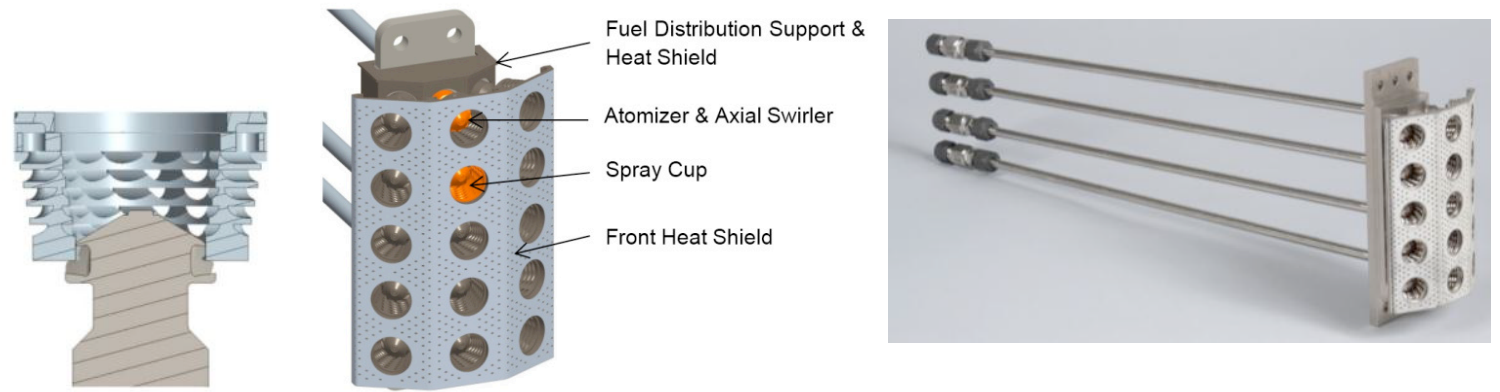

Fig. 2 The Parker Three-Zone Injector (3ZI) concept built for and tested under the NASA ERA program.

Table 1. Nominal experimental conditions.

\begin{tabular}{|c|c|c|c|}
\hline \multirow{2}{*}{$\begin{array}{c}\text { Inlet Pressure } \\
\text { (kPa) }\end{array}$} & \multirow{2}{*}{$\begin{array}{c}\text { Inlet } \\
\text { Temperature }\end{array}$} & \multicolumn{2}{|c|}{ Injector } \\
\cline { 3 - 4 } & (K) & 3ZI-1 & 3ZI-2 \\
\hline $\mathbf{7 1 0}$ & $\mathbf{5 7 0}$ & $\checkmark$ & \\
& $\mathbf{7 3 5}$ & $\checkmark$ & \\
\hline $\mathbf{1 , 0 3 4}$ & $\mathbf{5 7 0}$ & $\checkmark$ & $\checkmark$ \\
& $\mathbf{7 3 5}$ & $\checkmark$ & $\checkmark$ \\
\hline $\mathbf{1 , 3 7 9}$ & $\mathbf{8 2 7}$ & $\checkmark$ & $\checkmark$ \\
& $\mathbf{5 7 0}$ & $\checkmark$ & $\checkmark$ \\
& $\mathbf{7 3 5}$ & $\checkmark$ & $\checkmark$ \\
\hline $\mathbf{1 , 7 2 4}$ & $\mathbf{8 2 7}$ & $\checkmark$ & \\
& $\mathbf{5 7 0}$ & $\checkmark$ & \\
\hline & $\mathbf{7 3 5}$ & $\checkmark$ & \\
\hline
\end{tabular}

acquisition system. It recorded facility conditions such as temperature, pressures, and flow rates.

Dynamic pressure fluctuations were recorded using a Data Translation DT9841-sb high-speed data acquisition system. Dynamic data was recorded at 10 $\mathrm{kHz}$ for the 3ZI-1 injector and $20 \mathrm{kHz}$ for the $3 Z \mathrm{ZI}-2$ for a minimum of a 10 second period. Table 2 shows the type and model of the sensors, their location, and other details. The infinite probe measurements were compensated as discussed by Englund [17] and Samuelson [18]. Each probe consists of a $30 \mathrm{~m}$ coil of $6.35 \mathrm{~mm}$ outer diameter steel tubing. The probe tip consisted of $6.35 \mathrm{~mm}$ outer diameter steel tubing, $920 \mathrm{~mm}$ long, that isolated the transducer from the hot gases and thermal radiation effects. The probe's tip was 
mounted flushed with the flame tube wall. The lengths of the tip probe and the coil tubing were kept the same for each probe to minimize channel-to-channel variations. The $30 \mathrm{~m}$ coil is there to attenuate the acoustic waves in a way that

Table 2. Location and type of dynamic pressure transducers utilized during the experiments.

\begin{tabular}{|l|c|c|c|c|c|}
\cline { 2 - 6 } \multicolumn{1}{c|}{} & \multicolumn{4}{c|}{ Injector } \\
\cline { 2 - 6 } \multicolumn{1}{c|}{} & \multicolumn{3}{c|}{ 3ZI } \\
\hline Variable & $\mathrm{p}_{3}^{\prime}$ & $\mathrm{p}_{4}^{\prime}$ & $\mathrm{p}_{4}^{\prime}$ & $\mathrm{p}_{3}{ }_{3}$ & $\mathrm{p}_{4}^{\prime}$ \\
\hline Brand & $\mathrm{PCB}$ & DyTran & $\mathrm{PCB}$ & $\mathrm{PCB}$ & $\mathrm{PCB}$ \\
\hline Model & $112 \mathrm{~A} 22$ & $2200 \mathrm{~V} 1$ & $124 \mathrm{~A} 21$ & $112 \mathrm{~A} 22$ & $112 \mathrm{~A} 22$ \\
\hline Sensor stanoff, $\mathbf{c m}$ & 22 & 35 & 25 & 94 & 94 \\
\hline Axial location, $\mathbf{c m}$ & -32 & 25 & 25 & -32 & 25 \\
\hline Style & infinite tube & infinite tube & dead end & infinite tube & infinite tube \\
\hline
\end{tabular}

the amplitude of any reflected wave that returns to the transducer's face is well below the amplitude of the direct wave.

The coil and the probe tip tubing were constantly purged with nitrogen to prevent the hot air and combustion gases from entering the probe and damaging the transducer. An automatic valve was used to maintain the pressure inside the tubing higher than the flame tube pressure. The pressure upstream of the injector was used as the reference pressure since it is only a few psi higher than the mean pressure inside the flame tube. The valve was set up to supply an outlet pressure approximately $69 \mathrm{kPa}$ above the reference pressure.

The flame tube was instrumented with thermocouples to measure inlet and exhaust temperatures. Pressure transducers were used to measure inlet and exit pressures as well as pressure drops across the inlet venture, injector, flame tube, and exit. A calibrated venturi was used to measure air mass flow.

The exhaust emissions were measured utilizing a single probe with five sampling locations located at the same axial location as the downstream dynamic pressure sensors. During the experimental evaluation of the 3ZI-1 injector, it was found that at $\mathrm{P}_{3}=710 \mathrm{kPa}, \mathrm{T}_{3}=570 \mathrm{~K}$, and below a fuel air ratio of 0.035 , the $\mathrm{CO}$ emissions began to rise and the combustion efficiency dropped. It was concluded that the low injection pressure and the coarse fuel spray resulted in lower fuel vaporization and suggested that using a lower flownumber atomizer could improve the low inlet pressure, inlet temperature performance, i.e, the 3ZI-2 injector [4]. Figure 3 shows a comparison between the $\mathrm{NO}_{\mathrm{x}}$ emissions of the 3ZI-1 and 3ZI2 injectors for the same nominal conditions. Note that the data was plotted without corrections to the emissions due to deviation of the actual operating point from the nominal operating conditions. In general, reducing the flow number of the 3ZI-1 injector did not have a significant impact on $\mathrm{NO}_{\mathrm{x}}$, but what impact does it have on the combustion dynamic characteristics? It will be discussed in the next sections.

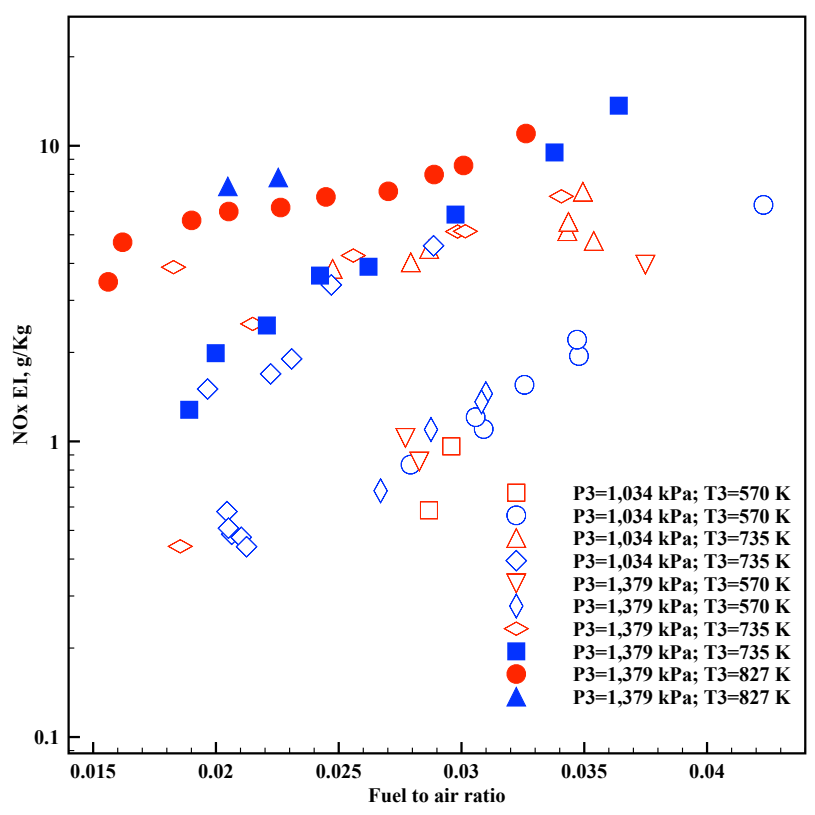

Fig. 3 Comparison between the NOx emissions of the 3ZI-1 injector (red) and the 3ZI-2 injector (blue). 


\section{Results and Discussion}

\section{3ZI-1 Injector}

The combustion dynamics of the 3ZI-1 and 3ZI-2 injector concepts were studied under the experimental conditions shown in Table 1. For the purpose of this paper, the flame tube will be considered unstable when the fluctuation amplitude is greater than $0.5 \%$ of the mean flame tube pressure and there is a defined peak.

The combustion dynamic characteristics of the 3ZI-1 injector were evaluated over the test conditions listed in Table 1. The combustion dynamics of the 3ZI-1 injector for the 710 and 1,034 kPa inlet conditions are shown in Figs. 4, and 5. The combustion dynamics for the other two inlet pressure conditions are not shown for brevity. The dynamic pressure spectra for the different conditions evaluated is similar with the exception of the magnitude. The effect of inlet air temperature on the dynamic response of the 3ZI-1 injector is clearly seen in those figures. Factors that enhance the chemical reaction rates will tend to reduce instabilities [19]. Increasing the inlet temperature reduces the evaporation time and the chemical reaction time. Thus, it should be expected that the dynamic pressure fluctuations
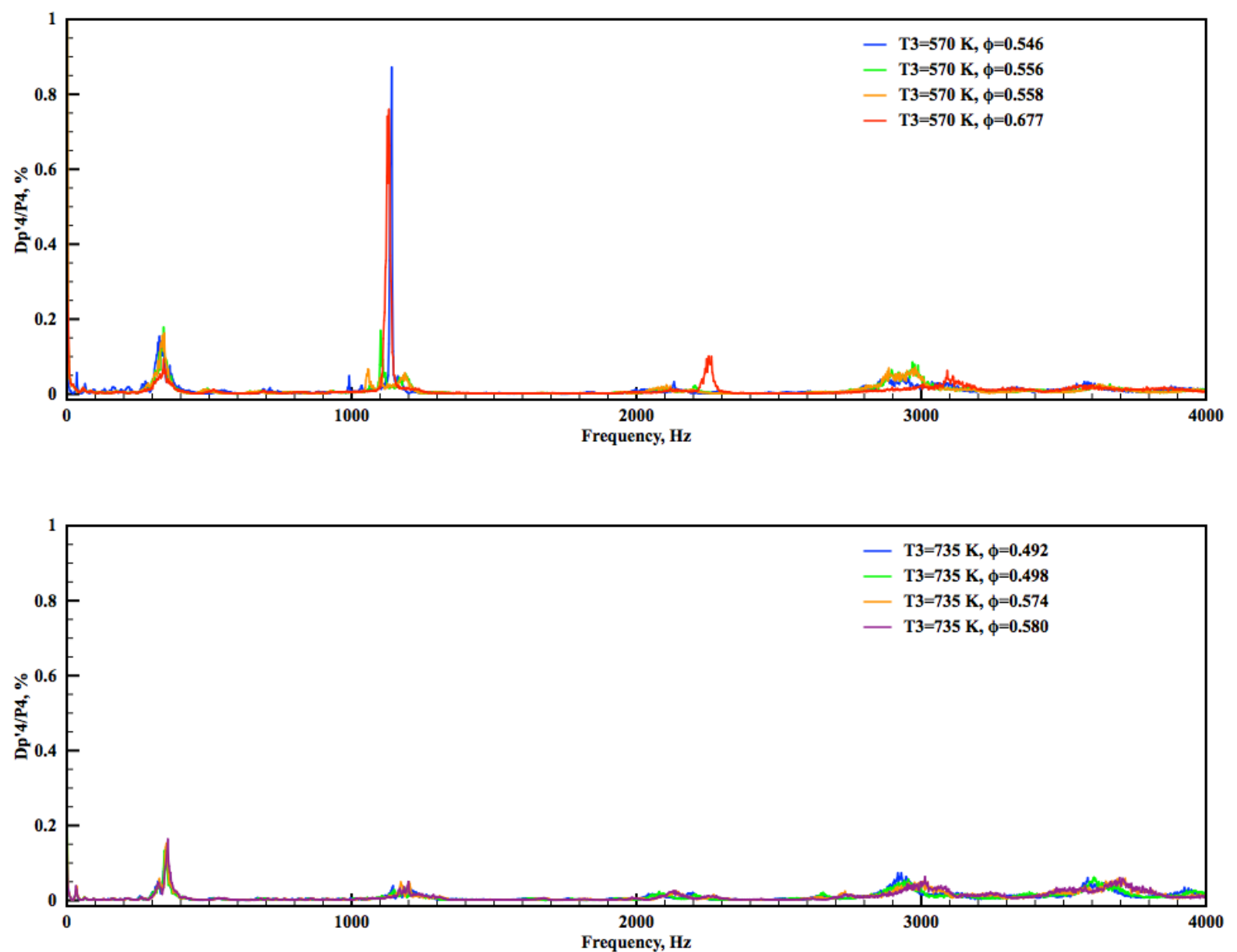

Fig. 4 Combustion dynamic characteristics of the 3ZI-1 injector at $710 \mathrm{kPa}$

magnitude for the higher inlet temperature conditions to be lower.

The figures also show that in general, the amplitude of the dynamic pressure fluctuations, increase as the equivalence ratio increases. This same behavior was reported in [15] for a research multi-point fuel injector. They identified some of the same factors that contribute to instability growth, like the phase relationship between the heat addition and the combustion chamber acoustics (Rayleigh criterion), vortex shedding, other turbulent phenomena generated by the fuel injectors, and the energy introduced into the combustor by burning 

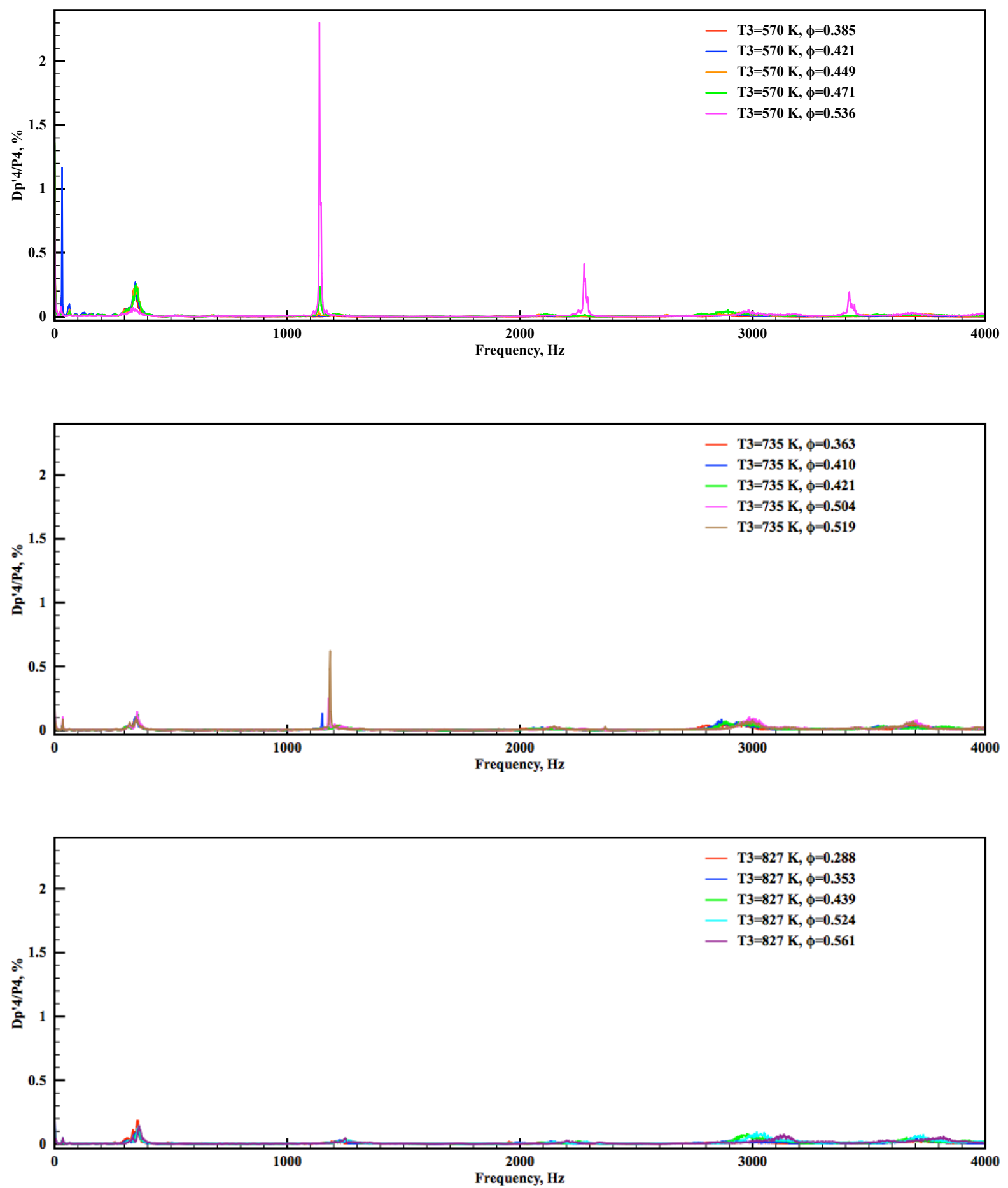

Fig. 5 Combustion dynamic characteristics of the 3ZI-1 injector at 1,034 kPa.

Utilizing the combustion instability criteria listed above, the 3ZI-1 injector exhibited unstable behavior at the following conditions: inlet pressure $710 \mathrm{kPa}$, inlet temperature $570 \mathrm{~K}$ and $\phi=0.546$ and 0.677 ; inlet pressure 1,034 $\mathrm{kPa}$, inlet temperature $570 \mathrm{~K}$, and $\phi=0.421$ and 0.536 ; and at an inlet pressure of $1,034 \mathrm{kPa}$, inlet temperature $570 \mathrm{~K}$, and $\phi=0.536$. Note that all fuel nozzles were flowing the same fuel flow rate and uniform throughout. The frequencies 
at which the peaks occurred are shown in Table 3. The fundamental frequency for the conditions shown in Figs. 3 and $4, f_{0}$, given by the following expression, $f_{0}=\mathrm{c} 2 L$, where $\mathrm{c}$ is the speed of sound, which is a function of the gas temperature, and $L$ is the flame tube length, is between 455 and $511 \mathrm{~Hz}$. The first harmonics will be 910 and 1,022 $\mathrm{Hz}$, respectively. Neither of those frequencies are near the measured values that ranged between 1,132 and 1,141 $\mathrm{Hz}$. Since this not a closed system, we looked at the possibility of the instability being a beat of the quarter frequency. As shown in Table 3, the measured peaks at inlet pressure $710 \mathrm{kPa}$, inlet temperature $570 \mathrm{~K}$, and $\phi=0.546$; inlet pressure $1,034 \mathrm{kPa}$, inlet temperature $570 \mathrm{~K}$, and $\phi=0.536$; and inlet pressure $710 \mathrm{kPa}$, inlet temperature $735 \mathrm{~K}$, and $\phi=0.519$, are very close to the $5^{\text {th }}$ harmonic of the quarter frequency. This may indicate the likelihood of an acoustic excited instability. The difference is larger at the $\phi=0.667$ suggesting a different exciting mechanism. Without direct optical access to the flame is very difficult to pinpoint the source of that oscillation. A possible source of acoustic excitation

Table 3. Measured versus calculated peak frequencies.

\begin{tabular}{|c|c|c|c|c|c|}
\hline $\begin{array}{c}\text { Inlet } \\
\text { Pressure } \\
(\mathrm{kPa})\end{array}$ & $\begin{array}{c}\text { Inlet } \\
\text { Temperature } \\
(\mathbf{K})\end{array}$ & $\phi$ & $\begin{array}{c}\text { Quarter } \\
\text { Frequency } \\
(\mathbf{H z})\end{array}$ & $\begin{array}{c}\text { 5th } \\
\text { Harmonic } \\
(\mathbf{H z})\end{array}$ & $\begin{array}{c}\text { Measured } \\
\text { Peak } \\
(\mathrm{Hz})\end{array}$ \\
\hline 710 & 570 & 0.546 & 232 & 1160 & 1141 \\
& 570 & 0.667 & 244.5 & 1222.5 & 1132 \\
\hline 1,034 & 570 & 0.421 & 217.5 & 1087.5 & 32 \\
& 570 & 0.536 & 230.5 & 1152.5 & 1138 \\
& 735 & 0.519 & 236 & 1180 & 1182 \\
\hline
\end{tabular}

at the measured frequencies could be another hydrodynamic mode, like the precessing vortex core.

At the $1,034 \mathrm{kPa}, 570 \mathrm{~K}$ and $\phi=0.421$ condition, there's an instability that occurred at around $32 \mathrm{~Hz}$. Lowfrequency instabilities have been referred to by different names i.e., "lean-limit", "cold tone", an "incipient blowout", "entropy wave" or "equivalence ratio oscillation." [13, 19, 20]. In a lean-limit instability study [13], high-speed movies were utilized to identify the physical mechanism causing the instability. It was observed that as the flame enters the shear layer, is locally extinguished and lifts up, the extinguished region fills with unburned reactants again, the flame flashbacks igniting the unburned reactants creating pressure rise and starting next cycle. That is very likely what is happening within this flame tube. It is also shown in Fig. 5 that as the inlet temperature increases the magnitude of the low frequency instabilities decreases possibly due to increased chemical reaction rates leading to less flameouts and re-ignition of the fuel/air mixture.

\section{3ZI-2 Injector}

The combustion dynamic characteristics of the 3ZI-2 injector were experimentally evaluated at the conditions shown in Table 1. Note that due to some hardware limitations, it was not possible to evaluate the 3ZI-2 injector at all the same conditions as the 3ZI-1 injector. As previously stated, the 3ZI-2 injector is the same injector as the 3ZI-1, except that the fuel flow number is half of the what the 3ZI-1 injector had. The hypothesis was the smaller flow number, i.e., orifice size, would lead to improved atomization, vaporization, and mixing to help further reduce NOx emissions.

At the conditions evaluated, the combustion dynamic characteristics were very low. There were no peaks greater than $0.5 \% \mathrm{Dp}^{\prime} / \mathrm{DP}$ present. Some small peaks were measured at the lower frequencies and those could be attributed of lean blowout occurring within the flame, as previously discussed. It could also be seen in Figs. 6 and 7, like for the 3ZI-1 injector, the magnitude of those peaks was reduced as the inlet temperature increased possibly due to increases in the chemical reaction rates due to better atomization.

In addition to the low frequency peaks, there were some peaks measured around the $1860 \mathrm{~Hz}$ frequency that were only present at both inlet pressures tested, but only at the $735 \mathrm{~K}$ inlet temperature condition. The peaks are not affected by the sonic velocity since there's no change in frequency as the gas temperature changes with changes in equivalence ratio. Again, a possible explanation could be a hydrodynamic mode that's excited, but only at $735 \mathrm{~K}$ inlet temperature. 

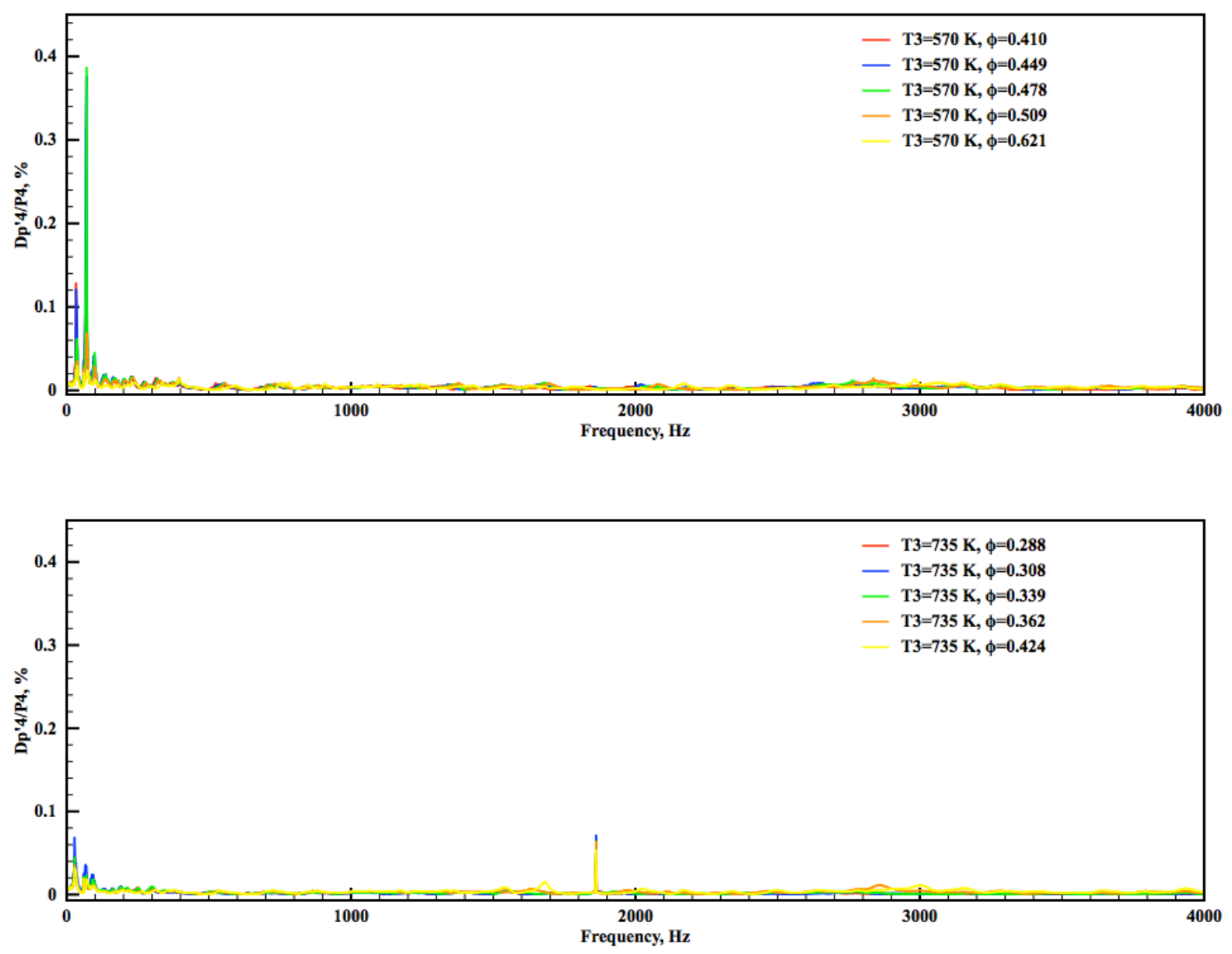

Fig. 6 Combustion dynamic characteristics of the 3ZI-2 injector at $1,034 \mathrm{kPa}$ 

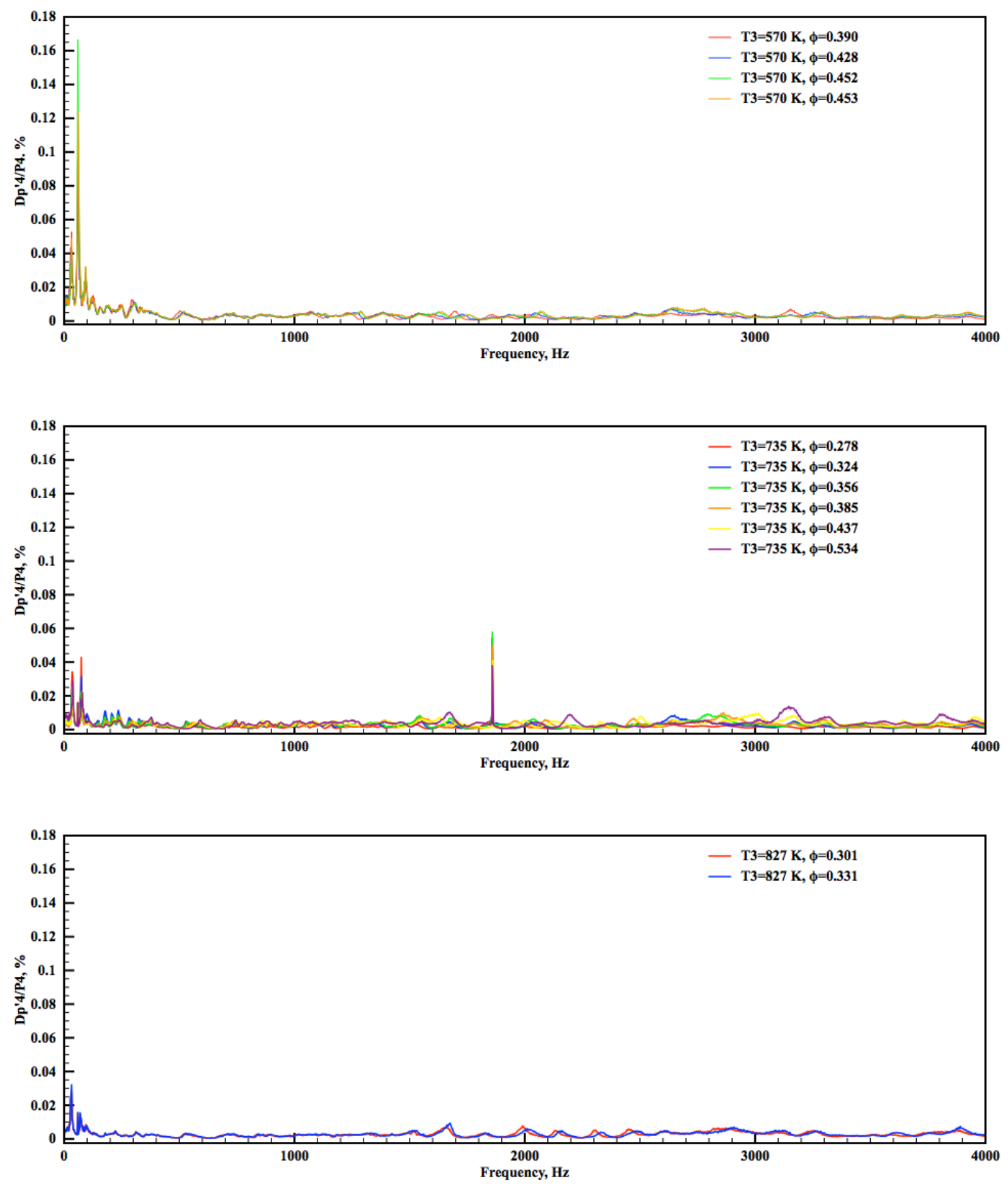

Fig. 7 Combustion dynamic characteristics of the 3ZI-2 injector at 1,379 $\mathrm{kPa}$ inlet pressure.

\section{E. 3ZI-1 injector vs the 3ZI-2 injector}

The 3ZI-1 and the 3ZI-2 injectors shared the same swirler design, but differ in the fuel flow number, the 3ZI-2 injector had a flow number that was half of the 3ZI-1's flow number. The objective of reducing the flow number was to improve atomization, which would lead to improved atomization, evaporation, and mixing and lower emissions. 
Figure 4 shows a comparison of the combustion dynamics between the 3ZI-1 and the 3ZI-2 injectors. The peaks around the $350 \mathrm{~Hz}$ for the 3ZI-1 injector are most likely a Helmholtz resonance inside the tube between the pressure transducer and flame tube.

The flow number of the 3ZI-2 injector was half that of the 3ZI-1 injector. This was accomplished by reducing the orifice size of the simplex injector. For the same fuel flow, the velocity of the fuel coming out is much higher than for the 3ZI-1 injector and the pressure drop across the nozzle is also higher. This change will produce a spray pattern with smaller droplets and deeper penetration into the flame tube. The finer droplets and its distribution along the flame tube will create a completely different flow field than that of the 3ZI-1 injector with different heat release rates, vortex shedding characteristics, and flame front location with its corresponding effect on the combustion dynamic characteristics. The data shown in Fig. 8 seems to indicate that reducing the flow number for the 3ZI-2 injector was able to reduce the magnitude of the dynamic pressures inside the flame tube to the point that no combustion instabilities were measured for the conditions that we were able to achieve with the 3ZI-2 injector. In general, the dynamic pressure fluctuations for the 3ZI-2 injector were much lower than the 3ZI-1 injector. An experimental investigation conducted to study of spray dynamics under thermoacoustic oscillations, found that acoustically unstable modes depended on the atomizing capacity of the fuel injectors and the injectors with the smaller droplet diameters were able to delay the transition to unstable regimes [21]. This seems to explain why the 3ZI-2 injector, under the conditions presented here,
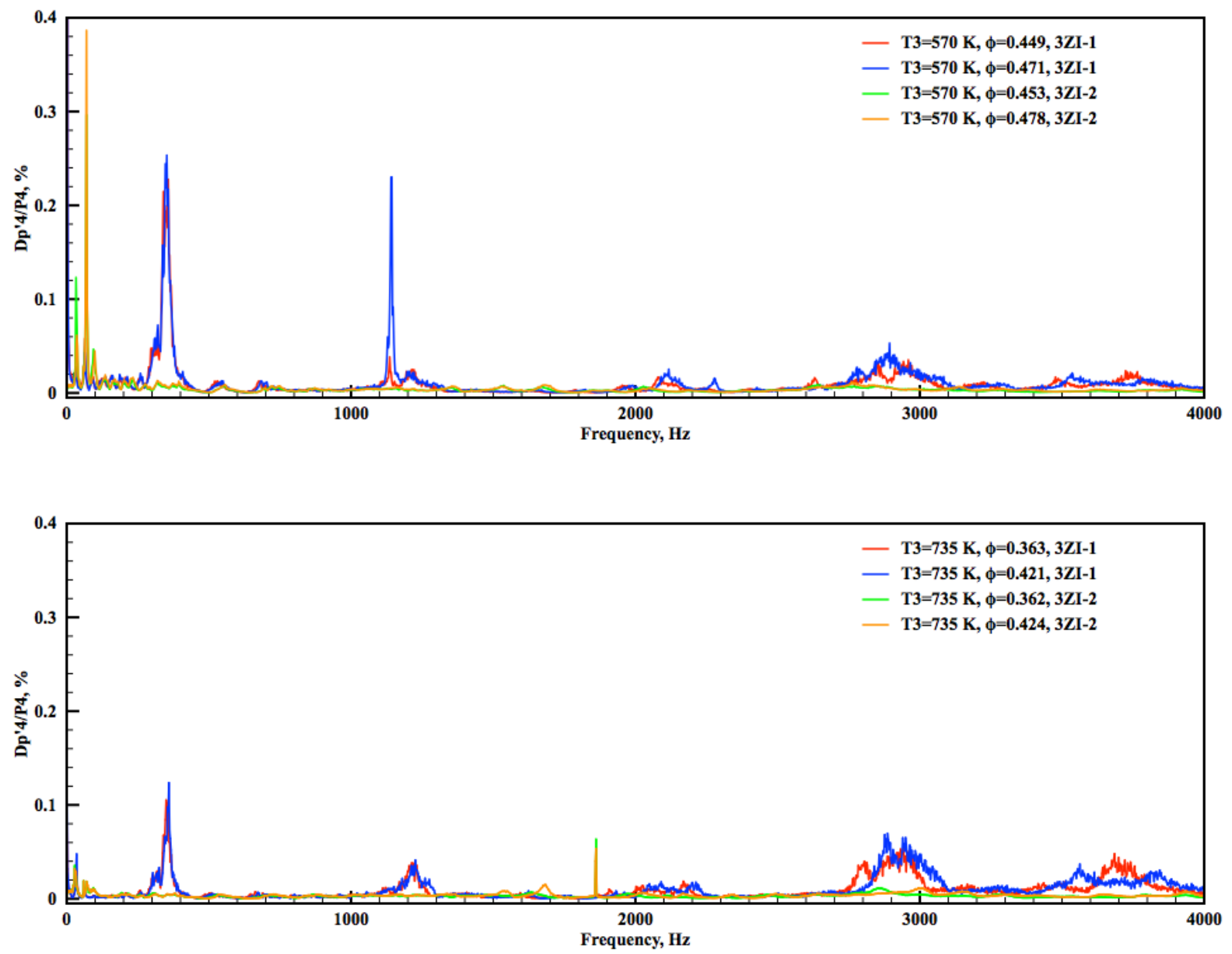

Fig. 8 Comparison of combustion dynamic characteristics at 1,034 kPa between the 3ZI-1 and 3ZI-2 injectors.

did not show any combustion instabilities. 


\section{F. Fuel Staging}

An objective for the 3ZI design was improve the low power operation over the previous design. The 3ZI concept approach to improve low power operation was the paneled arrangement that lead to formation of three flame zones, one per panel reducing coupling between zones to facilitate low power staged operation.

Figure 9 shows the combustion dynamic characteristics for the 3ZI-1 and 3ZI-2 injectors during fuel staged operation. Fuel staging means that only the center column was fueled. For the conditions shown in Fig. 9, all five cups in the center panel were flowing the same fuel amount, except for two conditions for the 3ZI-1 injector (top figure) where the pilot cup was flowing the same amount of fuel as the other 4 cups together. Running a rich equivalence pilot did not have any significant effect on the dynamic characteristics of the flame tube. Both injectors exhibit the same general combustion dynamic characteristics as when all fuel injectors were lit. The 3ZI-2 injector again had lower dynamic pressure fluctuations compared to the 3ZI-1.
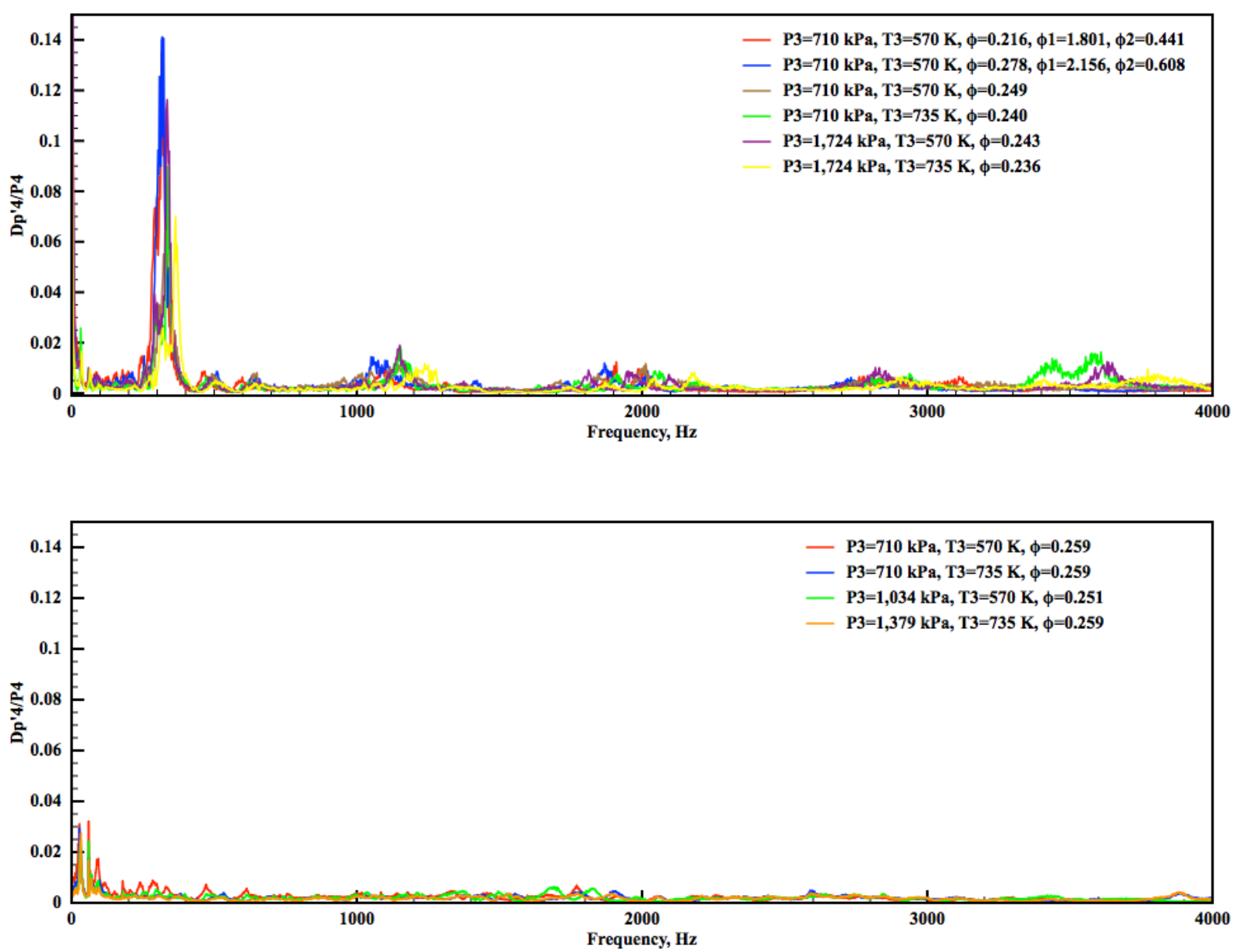

Fig. 9 Combustion dynamic characteristics for the 3ZI-1 (top) and 3ZI-2 (bottom) injector with only the center panel lit.

\section{Summary}

The combustion dynamics of two advanced, multi-cup injectors were evaluated over a range of inlet pressures up to $1,724 \mathrm{kPa}$, non-vitiated air temperatures up to $827 \mathrm{~K}$, and adiabatic flame temperatures up to $1975 \mathrm{~K}$. Dynamic pressure measurements indicated the presence of thermoacoustic instabilities at certain operating conditions. Those thermoacoustic instabilities were found to be tones of the quarter wave natural frequency of the flame tube. There was a particular instability that seemed to be the result of another source of excitation, potentially the PVC. Under a 
particular operating condition, a lean limit instability was found. The 3ZI-2 injector, with a flow number half of the 3ZI injector, did not exhibit any instabilities and in general the dynamic pressure fluctuations were lower than those of the 3ZI-1 injector for the same nominal operating conditions. Operating the 3ZI with only the center column lit did not excite any instabilities.

Reducing the flow number of the of the 3ZI-1 injector produced lower dynamic pressure fluctuations inside the flame tube and no instabilities. Changing the fuel spray characteristics, size, dispersion, and evaporation rate, affected the heat release rates, vortex shedding characteristics, the phase relationship between the heat addition and the combustion chamber acoustics, and flame front location in a way that created a less dynamic fluctuating pressure and acoustic environment inside the flame tube.

\section{Appendix}

During the evaluation of the 3ZI-1 injector we had two separate dynamic measurements for $\mathrm{p}^{\prime} 4$ as shown in Table 2 , the infinite probe sensor and the water-cooled sensor (dead end). Early on the test program the infinite probe sensor failed and the test continued with the water-cooled sensor only. Figure A1 shows a direct comparison between the infinite probe sensor and the water cooled. As shown in the figure, both sensors were able to capture the combustion dynamics characteristics of the injector with high confidence that the results between the 3ZI-1 and the 3ZI-2 can be compared directly since the 3ZI-2 results were obtained using an infinite probe sensor. The results presented for the 3ZI-1 injector in this paper will be using the water-cooled pressure sensor. The peak shown for the water-cooled probe

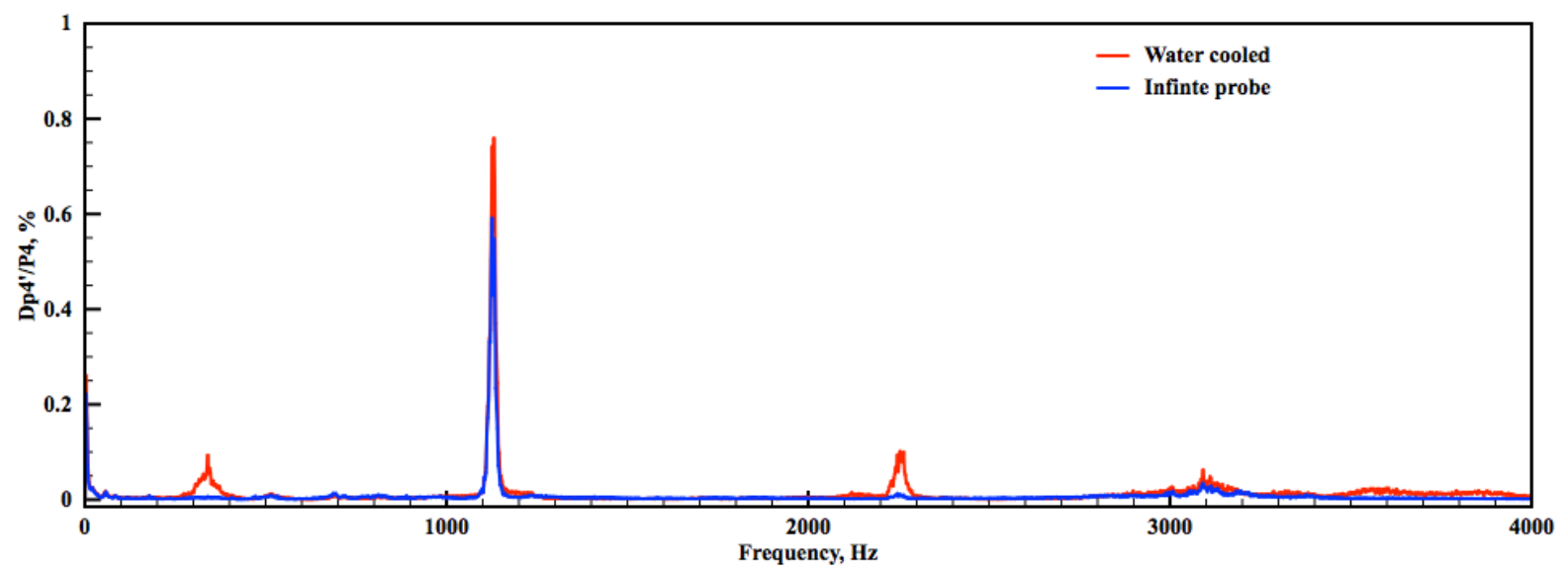

Fig. A1 Comparison between the water-cooled probe and the infinite probe, $\mathrm{P3}=710 \mathrm{kPa}, \mathrm{T3}=570 \mathrm{~K}$, and $\phi=0.667$

is most likely a Helmholtz tone within the cavity of the tube at the end of the water-cooled pressure sensor.

\section{Acknowledgments}

This work was sponsored by the Advanced Air Transportation Technology (AATT) Project within NASA's Advanced Air Vehicles Program.

\section{References}

[1] R. Tacina, P. Lee, and C. Wey, A Lean-Direct-Injection Combustor Using a 9 Point Swirl Venturi Fuel Injector, ISABE-2005-1106 Report No., 2005.

[2] R. Tacina, C. Wey, P. Laing, and A. Mansour, A Low-NOx Lean-Direct Injection, MultiPoint Integrated Module Combustor Concept for Advanced Aircraft Gas Turbines, NASA/TM-2002-211347 Report No., 2005.

[3] R. Tacina, C.-P. Mao, and C. Wey, Experimental Investigation of a Multiplex Fuel Injector Module with Discrete Jet Swirlers for Low Emissions Combustors, AIAA-2004-0135 Report No., 2004

[4] Steinthorsson E, Mansour A, Hollon B, Teter M, Chang C. "Advanced Multi-Cup Fuel Injector Technology for Environmentally Responsible Aviation Gas Turbine Engines," ASME Paper No. GT2015-42703

[5] Rayleigh, J.W.S., "The Theory of Sound," Vol. 2, 1896, Macmillan and Co.

[6] Lilly, D. G., "Swirling Flow in Combustion: a Review," AIAA J., Vol. 15, No. 8, 1977, pp. 1063-1078 
[7] Huang, Y., and Yang, V., 2009, "Dynamics and Stability of Lean-Premixed Swirl-Stabilized Combustion," Prog. Energy Combust. Sci., 35(4), pp. 293-364.

[8] Ducruix, S., Schuller, T., Durox, D., and Candel, S., 2003, "Combustion Dynamics and Instabilities: ElementaryCoupling and Driving Mechanisms," J. Propul. Power, 19(5), pp. 722-734.

[9] Candel, S., Durox, D., Schuller, T., Bourgouin, J.-F., and Moeck, J. P., 2014, "Dynamics of Swirling Flames," Annual Review of Fluid Mechanics, 46(1), pp. 147-173.

[10] Yi, T., Santavicca, D. A., "Flame Spectra of a Turbulent Liquid-Fueled Swirl-Stabilized LDI Combustor," in 47th AIAA Aerospace Sciences Meeting including The New Horizons Forum and Aerospace Exposition. 2009, American Institute of Aeronautics and Astronautics.

[11] Yi, T., Santavicca, D. A., "Combustion Instability in a Turbulent Liquid-Fueled Swirl-Stabilized LDI Combustor," in 45th AIAA/ASME/SAE/ASEE Joint Propulsion Conference \& Exhibit. 2009, American Institute of Aeronautics and Astronautics.

[12] Huang, C., Gejji, R., and Anderson, W. E., "Combustion Dynamics Behavior in a Single-Element Lean Direct Injection (LDI) Gas Turbine Combustor," AIAA 2014-3433, 2014.

[13] Dhanuka, S.K., Temme, J.E., Driscoll, J.F., "Lean-limit combustion instabilities of a lean premixed prevaporized gas turbine combustor" Proc. Combust. Inst., Vol. 33, 2011, pp. 2961-2966.

[14] Tacina, K. M., Chang, C.T., Lee, P., Mongia, H., Podboy, D. P., and Dam, B., "Assessment of Combustion Dynamics in a Second-Generation Swirl-Venturi Lean Direct Injection Combustion Concept," ISABE-2015-20249

[15] Acosta, W. A., Chang, C. T., "Experimental Combustion Dynamics Behavior of a Multi-Element Lean Direct Injection (LDI) Gas Turbine Combustor, AIAA 2016-4589 Report No., 2016

[16] Tacina, K. M., Hicks, Y. R., "A comparison of combustion dynamics for multiple 7-point lean direct injection combustor configurations," $10^{\text {th }}$ U.S. National Combustion Meeting, April 23-26, 2017, College Park, MD

[17] Englund, D. R. and Richards, W. B, "The Infinite Line Pressure Probe,” NASA TM-83582, 1984

[18] Samuelson, R. D., "Pneumatic Instrumentation Lines and Their Use in Measuring Rocket Nozzle Pressure," Report No. RN-DR-0124, Nuclear Rocket Operations, Aerojet-General Corporation, 1967.

[19] B.T. Zinn, T.C. Lieuwen, in: T.C. Lieuwen, V. Yang (Eds.), AIAA Progress in Astronautics and Aeronautics, vol. 210, 2005, pp. 3-26 (Chapter 1).

[20] Temme, J.E., Allison, P.M., Driscoll, J.F., "Combustion instability of a lean premixed prevaporized gas turbine combustor studied using phase-averaged PIV," Combustion and Flame 161 (2014) 958-970.

[21] Chishty, W. A, Lepera, S. D., Vandsbuger, U., "Spray Combustion Dynamics under Thermoacoustic Oscillations," International Journal of Mechanical and Mechatronics Engineering, Vol. 5, No. 1, 2011 